\title{
DOS PASOS ADELANTE Y UNO ATRÁS: PROTECCIÓN DE LOS DERECHOS DE LAS MUJERES EN LA JURISPRUDENCIA CONSTITUCIONAL COLOMBIANA DE 2018
}

Two steps forward and one back: Protection of the women's rights in the Colombian Constitutional jurisprudence of 2018

\author{
PAULA ROBLEDO SILVA ${ }^{\top}$ \\ Universidad Externado de Colombia \\ paula.robledo@uexternado.edu.co \\ MARIANA MEDINA BARRAGÁN² \\ Universidad Nacional de Colombia \\ mmedinaba@unal.edu.co \\ Cómo citar/Citation \\ Robledo Silva. P. y Medina Barragán, M. (2019). \\ Dos pasos adelante y uno atrás: protección de los derechos de las mujeres \\ en la jurisprudencia constitucional colombiana de 2018. \\ Anuario Iberoamericano de Justicia Constitucional, 23(2), 54 1-558. \\ doi: https://doi.org/10.18042/cepc/aiic.23.18
}

\section{SUMARIO}

I. INTRODUCCIÓN. II. DOS PASOS ADELANTE: EL MANTENIMIENTO DE LA PROTECCIÓN OTORGADA A LA INTERRUPCIÓN VOLUNTARIA DEL EMBARAZO Y LA EXENCIÓN TRIBUTARIA A LA HIGIENE MENSTRUAL: 1. El mantenimiento de la protección otorgada a la interrupción voluntaria del embarazo. 2. La exención tributaria a la higiene menstrual. III. UNO ATRÁS: EL CAMBIO DE PRECEDENTE SOBRE ESTABILIDAD LABORAL REFORZADA A MUJERES GESTANTES. IV. CONCLUSIONES. APÉNDICE LEGIILATIVO. BIBLIOGRAFíA.

1 Docente e investigadora de la Universidad Externado de Colombia y defensora delegada para los Asuntos Constitucionales y Legales de la Defensoría del Pueblo de Colombia. Número ORCID: 0000-0002-7950-5233.

2 Abogada y magíster en Derecho Constitucional de la Universidad Nacional de Colombia. Actualmente, asesora de la Defensoría Delegada para los Asuntos Constitucionales y Legales de la Defensoría del Pueblo de Colombia. 


\section{INTRODUCCIÓN}

La Constitución Política de 1991 constituyó un cambio de paradigma político, económico y jurídico que, entre otros asuntos, representó la posibilidad de avanzar en la consecución de la igualdad material de poblaciones históricamente discriminadas, al consagrar la obligación de superar las razones en las que se sustenta su exclusión.

Así, la igualdad fue establecida como valor, principio y derecho fundamental, lo cual, de acuerdo con la jurisprudencia constitucional, trasciende la concepción formal de esta, para comprender que su verdadera dimensión implica la obligación de adoptar medidas dirigidas a la promoción de una igualdad real y efectiva, en particular, a favor de grupos marginados y de quienes se encuentran en circunstancias de debilidad manifiesta.

En este propósito, los pronunciamientos de la Corte Constitucional han sido fundamentales, pues, a través del control abstracto de constitucionalidad, el ordenamiento jurídico se ha ajustado progresivamente al reconocimiento, inclusión y respeto de estas personas; y en el estudio de casos concretos, las órdenes dictadas configuran trascendentales logros para su protección.

Lo anterior ha tenido un efecto significativo sobre la vida de las mujeres y niñas, pues las diversas formas de violencia que sufren cotidianamente y los múltiples obstáculos enfrentados para la garantía y goce de sus derechos han sido el centro de la discusión de numerosas sentencias del Alto Tribunal. La mayoría de sus decisiones al respecto son una victoria determinante en la lucha por erradicar las causas y consecuencias de estas graves problemáticas, aun cuando subsistan inmensos retos en la materia.

Específicamente, durante 2018, fueron proferidos más de treinta fallos relacionados con violencia doméstica, sexual e institucional ${ }^{3}$. Entre ellos, en esta crónica jurisprudencial queremos destacar tres debido a su impacto en los derechos reproductivos de las mujeres y niñas. Se trata del que mantuvo más de una década de protección a la interrupción voluntaria del embarazo (SU-096 de 2018), el relativo a la exención tributaria a los elementos para la higiene menstrual (C-117 de 2018), y uno que inusitadamente dio lugar al retroceso a la estabilidad laboral reforzada de mujeres gestantes (SU-075 de 2018).

3 A partir de la sentencia T-735 de 2017, la Corte Constitucional indicó que «las autoridades encargadas de la atención de las mujeres víctimas de violencia de género incurren en violencia institucional cuando con su acción u omisión les causan o amenazan con causarles daño psicológico». En 2018 fue proferida la sentencia T-338 en la que se ordenó capacitar a todos los jueces y juezas de familia en género con el fin de evitar la violencia institucional. 
Dichos fallos evidencian que el progreso en la garantía de los derechos de la población femenina sigue siendo un proceso lento, cuya consolidación representa un desafío constante para la jurisprudencia constitucional, la cual, caminando hacia la igualdad, el año pasado, dio dos pasos adelante y uno atrás.

\section{DOS PASOS ADELANTE: EL MANTENIMIENTO DE LA PROTECCIÓN OTORGADA A LA INTERRUPCIÓN VOLUNTARIA DEL EMBARAZO Y LA EXENCIÓN TRIBUTARIA A LA HIGIENE MENSTRUAL}

\section{EL MANTENIMIENTO DE LA PROTECCIÓN OTORGADA A LA INTERRUPCIÓN VOLUNTARIA DEL EMBARAZO}

En la sentencia SU-098 de 2018, la Corte Constitucional revisó la tutela interpuesta por una mujer en avanzado estado de gestación quien consideró vulnerados sus derechos fundamentales a la vida, salud, dignidad y autonomía reproductiva, debido a la imposición de barreras por parte de su entidad prestadora de salud (EPS) para practicarle la interrupción voluntaria del embarazo (IVE) pese a haber acreditado dos de las causales estipuladas para el ejercicio de este derecho fundamental en la C-355 de 2006 ${ }^{4}$. Durante el trámite de la acción, el juez de primera instancia ordenó la realización del procedimiento a través de una medida provisional, pues estimó que, al contar con más de 25 semanas de embarazo y existir los certificados médicos pertinentes, correspondía, incluso antes de emitir el fallo de fondo, adoptar medidas urgentes a fin de evitar la consumación de la violación a los derechos invocados.

En el proceso se probó que había sido diagnosticada una enfermedad incompatible con la vida del feto y que la situación implicó también una afectación que ponía en riesgo la salud mental de la mujer. Los médicos certificaron estas circunstancias e incluso recomendaron llevar a cabo la IVE. A pesar de que se realizaron los trámites correspondientes para ello, las instituciones

\footnotetext{
4 En esta providencia se despenalizó el aborto en tres circunstancias especiales: 1) cuando la continuación del embarazo constituya peligro para la vida o la salud de la mujer, certificada por un médico; 2) cuando exista grave malformación del feto que haga inviable su vida, certificada por un médico, y 3i) cuando el embarazo sea el resultado de una conducta, debidamente denunciada, constitutiva de acceso carnal o acto sexual sin consentimiento, abusivo o de inseminación artificial o transferencia de óvulo fecundado no consentidas, o de incesto.
} 
del sector salud involucradas lo dilataron, incrementando las dificultades del procedimiento, pues debido al aumento de la etapa gestacional existen mayores riesgos para la vida y salud de la mujer, y se necesitan condiciones técnicas particulares para su práctica.

A partir de decenas de casos similares, el Alto Tribunal ha constatado los obstáculos enfrentados por la población femenina para el ejercicio del derecho a la interrupción voluntaria del embarazo y a partir de su análisis ha definido con claridad el contenido y alcance de este derecho fundamental. No obstante, este pronunciamiento convocó la atención nacional debido a que diversos medios de comunicación alertaron sobre la posibilidad de que modificara las condiciones de salvaguarda a la IVE en el país. En efecto, la primera ponencia presentada generaba variaciones al precedente jurisprudencial en la materia ${ }^{5}$, la cual no fue acogida por la Sala Plena, que decidió asignar la tarea de proyectar el fallo a otro magistrado para que plasmara la decisión mayoritaria de mantener los estándares de protección al derecho fundamental.

Con dicho propósito, la sentencia hizo énfasis en la trascendencia de la garantía de los derechos sexuales y reproductivos para el goce de la libertad, autonomía e la igualdad de las mujeres. Así, explicó que esos derechos «comparten su fundamento normativo y filosófico, pues se enmarcan en el empeño de avanzar en la eliminación de los estereotipos de género que han facilitado la discriminación histórica de la mujer, y en las reivindicaciones relativas a su libertad y autonomía sexual y reproductiva».

De esta manera, al ser la interrupción voluntaria del embarazo parte de los derechos reproductivos, se "compromete en su respeto y realización a todos los servidores y órganos del Estado, a los prestadores públicos y privados de seguridad social y a los particulares».

Debido a las características de la situación analizada, la Corte consideró necesario recordar que todos los asuntos relativos a la IVE tienen carácter urgente, pues a medida que avanza la gestación el procedimiento se hace más complejo y peligroso para la mujer involucrada. Esto tiene correspondencia con los patrones probatorios débiles o flexibles adoptados en la sentencia C-355 de 2006 para acreditar la materialización de cada una de las tres causales, pues disponer unos muy estrictos resultaría contraproducente para la protección del derecho a la IVE, ya que ello podría inhibir a los profesionales de la salud para certificar la necesidad del procedimiento debido a la penalización residual del aborto en nuestro ordenamiento jurídico.

5 El salvamento de voto a la sentencia SU-098 de 2018 presentado por la magistrada Cristina Pardo Schlesinger (2018) da cuenta del sentido en el que se orientaba el fallo. 
En el breve recuento de los estándares de protección del derecho a la IVE, fueron concretados algunos de estos con miras a materializar el goce efectivo de los derechos reproductivos de las mujeres gestantes en el país. Así, vale la pena destacar que, frente a la causal referida a grave malformación del feto que lo haga inviable con la vida, el Alto Tribunal precisó que esta no alude en modo alguno a los denominados «aborto eugenésico» o "aborto selectivo por discapacidad», pues su propósito no es otro que aliviar la angustia y padecimientos de la mujer que aloja en su cuerpo un feto que probablemente no vivirá, ya que obligarle a continuar la gestación en esas condiciones resulta desproporcionado e implica un trato cruel e inhumano que afecta su dignidad humana.

Igualmente, estimó pertinente aclarar que la determinación del grado de «probabilidad» de incompatibilidad con la vida no puede de ninguna manera derivar en una exigencia de certeza al respecto, y que su determinación nunca corresponde al juez constitucional, pues ello es competencia exclusiva de los profesionales de la salud tratantes de la mujer gestante.

La Corte también indicó que el derecho a la IVE no se agota en la realización de un procedimiento médico debido a que este involucra otros componentes de accesibilidad y disponibilidad que deben ser garantizados para su efectivo goce e hizo énfasis en que la conclusión según la cual la prohibición absoluta del aborto es violatoria de los derechos constitucionales de las mujeres llevada a cabo hace doce años implicó, al mismo tiempo, «el reconocimiento de que la interrupción voluntaria del embarazo, en los tres casos identificados por la Corte, integra el contenido ius fundamental de los postulados superiores comprometidos».

Finalmente, destacó que las medidas provisionales en el marco de la acción de tutela no tienen límites fijos. Estos dependen de las circunstancias particulares de cada caso, por lo cual, deben guiarse por el daño que se pretenda evitar. En ese sentido, la autoridad judicial se encuentra facultada para que en casos de extrema gravedad y urgencia se adopten decisiones no reversibles.

Teniendo en cuenta lo anterior, el Alto Tribunal confirmó la decisión adoptada en primera instancia, exhortó al Congreso de la República para que legisle acerca de la interrupción voluntaria del embarazo, «avanzando en la protección de los derechos fundamentales de las mujeres y buscando eliminar las barreras aún existentes para el acceso a la IVE», y ordenó al Ministerio de Salud y Protección Social que emita una regulación única en la cual se garantice este derecho en los casos despenalizados en la sentencia C-355 de 2006, con base en las reglas extraídas de la jurisprudencia constitucional, incluyendo sanciones frente a su incumplimiento.

El estudio de este caso dio cuenta de lo gravosos que pueden resultar los obstáculos para el ejercicio del derecho fundamental a la interrupción volun- 
taria del embarazo y la posibilidad de contar con una vida digna para las mujeres y niñas, en particular cuando se está en presencia de etapas gestacionales avanzadas, pues en estos casos las acciones diligentes y prontas son determinantes para evitar lesionar el conjunto de los derechos fundamentales de la persona involucrada, lo cual lamentablemente no está ocurriendo en el país ${ }^{6}$.

La propia Corte ha evidenciado previamente la existencia de barreras que dificultan o hacen nugatorio el derecho a la práctica de la interrupción voluntaria del embarazo de las mujeres gestantes incursas en una de las causales introducidas en la sentencia C-355 de 2006, y por ello ha tenido que insistir en que los requisitos para proceder conforme a la voluntad de aquellas son mínimos y muy claros, pues, por un lado, buscan proteger su dignidad, y, por otro, parten de reconocer que permitir el avance del estado de gestación al imponer trabas injustificadas es inadmisible, ya que se agrava la vulneración de los derechos fundamentales de las implicadas.

En aquellos casos en los que el período de gestación presenta una evolución avanzada, como el analizado en esta sentencia, se han generado inquietudes y resistencias al ejercicio del derecho fundamental por parte de distintos sectores, los cuales proponen el establecimiento de un término determinado para que pueda practicarse el procedimiento requerido, acorde con la posibilidad de vida extrauterina del feto. Ello constituye un error por representar una barrera adicional a las ya padecidas por las mujeres y niñas para hacer valer su deseo cuando, estando incursas en las causales, optan por la interrupción de su embarazo, y porque parte de la idea de concebir a la mujer solamente como un medio para el nacimiento del nuevo ser sin reparar en su dignidad ${ }^{7}$.

Desafortunadamente esto ocurrió en el año 2014 en el que la Corte Constitucional, mediante la sentencia T-532, introdujo un elemento diferente a los señalados en los pronunciamientos precedentes, derivado, según nuestro criterio, de una incorrecta interpretación del contenido de dicha garantía fundamental.

Lo anterior, por considerar que, en virtud del criterio médico, podría justificarse la negativa a practicar la interrupción voluntaria del embarazo, atendiendo a la etapa gestacional del mismo, por cuanto «en el caso de embarazos con estados de gestación muy avanzados, se está frente a fetos desarrollados que, en principio, tendrían posibilidades de sobrevivir de manera autónoma por fuera del vientre de la mujer».

Al respecto, véase La Mesa por la Vida y la Salud de las Mujeres (2017).

7 Esta posición fue sostenida por la Defensoría del Pueblo en la intervención presentada ante la Corte Constitucional cuando esta adelantaba el estudio del expediente T-6.612.909. 
Establecer que cuando la edad gestacional es tal que el desarrollo del feto permite que el mismo pueda sobrevivir a la vida extrauterina no existe obligación de proceder a la interrupción voluntaria del embarazo, aun cuando se encuentren configurados todos los presupuestos establecidos por la Corte para el efecto, constituye una limitación genérica al derecho reconocido en la sentencia C-355 de 2006 y, por tanto, viola el contenido esencial del mismo.

Contrarias a ese planteamiento, las experiencias de otros países que han tendido a conjugar elementos del sistema de causales y plazos lo han hecho para agregar a la restricción de los tiempos de gestación establecidos las causales, y no para limitar estas últimas a partir de un número de semanas determinadas y la determinación de viabilidad de vida extrauterina, ya que ello es violatorio del principio de no regresividad en el derecho a la salud, pues desconoce la imposibilidad técnica de realizar diagnósticos en ciertos momentos, no se corresponde con las barreras que han sufrido las mujeres en el país para ejercer el derecho fundamental a la interrupción voluntaria del embarazo - por las cuales han sido necesarios los pronunciamientos de la Corte Constitucional- y retrocedería en la protección del derecho a la salud y de los derechos sexuales y reproductivos de esta población.

Frente a la sentencia SU-096 de 2018, finalmente, es necesario mencionar que las órdenes dirigidas al Ministerio de Salud suponen un inmenso reto para esta entidad, pues el Consejo de Estado en un fallo de 2016 anuló algunas de las instrucciones de la Circular No. 003 de 2013 de la Superintendencia Nacional de Salud, mediante la cual se imparten instrucciones sobre la IVE, en aplicación de la Constitución Política, los tratados internaciones y las sentencias de la Corte Constitucional, tras considerar que estas últimas no pueden ser el fundamento para la regulación de un derecho fundamental ${ }^{8}$, tal como ocurre en este caso también.

En esa providencia, la Alta Corporación no solo desconoció el carácter vinculante de la jurisprudencia constitucional en la que se fundamentó la $\mathrm{Su}$ perintendencia para dictar las instrucciones correspondientes — con el fin de que los prestadores de salud adoptaran las medidas adecuadas para el ejercicio del derecho fundamental a la IVE por parte de las mujeres y niñas-, y que ordenó reiteradamente a dicha entidad adoptar este tipo de actos, sino que incluso evidenció su incomprensión de la sentencia C-355 de 2006, al afirmar, por ejemplo, que fueron sentencias de revisión de tutela las que prohibieron la objeción de conciencia institucional, cuando fue dicha providencia de control de constitucionalidad la que expresamente así lo hizo. A partir de

8 Consejo de Estado. Sala de lo Contencioso Administrativo. Sección Primera. Radicación número: 11001-03-24-000-2013-00257-00. 13 de octubre de 2016. 
esto anuló importantes instrucciones, dificultando con ello que la población femenina del país pueda acudir al sistema de salud para realizarse el procedimiento cuando se encuentra facultada para esto por estar incursa en alguna de las causales previstas por la jurisprudencia constitucional.

Así pues, teniendo en cuenta las múltiples barreras que enfrentan las mujeres y niñas para el ejercicio de este derecho fundamental luego de doce años de proferida la sentencia en que se despenaliza parcialmente el aborto en el país, mantener la protección a la IVE y ordenar su regulación, a fin de contribuir en su garantía, constituye un paso adelante para su materialización. Sin duda, avanzar en los derechos reproductivos de las mujeres permitirá que estas cuenten con mejores condiciones de vida, lo que tiene un efecto positivo en el desarrollo del conjunto de la población. Evitar que cientos de mujeres y niñas pongan en riesgo su salud, integridad e incluso su vida a diario para dar por terminadas gestaciones que acorde con la jurisprudencia constitucional deben ser atendidas dignamente por el sistema de salud es un problema de salud pública que debe convocar a la sociedad en su conjunto.

\section{LA EXENCIÓN TRIBUTARIA A LA HIGIENE MENSTRUAL}

En la sentencia C-117 de 2018, la Corte Constitucional estudió la demanda interpuesta en contra del aparte del art. 185 de Ley 1819 de 2016 que disponía gravar con el $5 \%$ del impuesto sobre las ventas (IVA) a las compresas y tampones higiénicos. En esta oportunidad, le correspondió determinar si la norma acusada vulneraba los principios de igualdad, equidad y progresividad tributarias al imponer el cobro del IVA a bienes utilizados únicamente por la población femenina, sin tener en cuenta su capacidad económica, y si además ello resultaba discriminatorio, por tratarse de elementos utilizados exclusivamente por mujeres y niñas para atender parte de las manifestaciones de un proceso biológico inevitable propio de las mismas, como lo es el período menstrual.

Con ese propósito, la sentencia recordó que si bien existe un amplio margen del legislador para determinar los tributos, también se han establecido claros límites a su poder a fin de garantizar que estos cumplan con su propósito y no afecten los derechos fundamentales de la población? . A partir de lo

9 Entre estos se encuentran el reconocimiento de Colombia como Estado social de derecho, la exigibilidad de los derechos sociales y económicos — así como la consecuente prohibición de regresividad-, los derechos a la alimentación y al mínimo vital y los principios del sistema tributario mencionados. 
anterior señaló que «el gravamen indiscriminado del IVA $^{10}$ relativo a todos los servicios o bienes, sin tener en cuenta su naturaleza, objetivos y finalidades y sin medir sus consecuencias sociales, puede violar el derecho a la igualdad», y destacó que la política tributaria "como factor determinante que moldea las relaciones con el Estado y que incide directamente en el desarrollo económico de las mujeres, puede reproducir patrones discriminatorios al reflejar las estructuras de poder sociales».

Así pues, atendiendo al objeto de la controversia, la Corte llamó la atención frente al hecho de que "la efectividad del derecho a la igualdad material de las mujeres es un imperativo constitucional, que es correlativo a la eliminación de todas las formas de discriminación contra las mujeres». En ese sentido, corresponde al Estado, por un lado, adoptar políticas públicas que se dirijan a superar los obstáculos enfrentados por esta población para conseguirla y, por otro, en el estudio de los eventos en los que se alega la existencia de discriminación en su contra debido a una circunstancia específica, verificar tanto el contexto y los diferentes factores que puedan contribuir o determinar la situación como el impacto que tiene la medida, desde una perspectiva interseccional ${ }^{11}$.

A partir de lo anterior, este pronunciamiento propuso una metodología que combina el análisis de la discriminación indirecta con el de la discriminación interseccional para el estudio del tributo cuestionado. La primera, referida a los tratamientos aparentemente neutros de los que se desprenden consecuencias fácticas desiguales para algunas personas limitando el goce efectivo de sus derechos ${ }^{12}$, y la segunda, consistente en el reconocimiento de que la confluencia de distintas categorías en una persona o grupo pueden acentuar una situación de discriminación, como ocurre con la orientación

10 El IVA como impuesto indirecto no parte de la identificación concreta y previa del sujeto contribuyente, y por tanto solo puede determinar su capacidad de pago a partir de la propensión al consumo de los bienes gravados con el tributo. En este sentido, debería aplicarse a los contribuyentes que con mayor frecuencia incurran en el hecho gravado y, por tanto, les corresponda pagar proporcionalmente más que los demás. Corte Constitucional. Sentencia C-117 de 2018.

12 «Se compone de dos criterios: Primero, la existencia de una medida o una práctica que se aplica a todos de manera aparentemente neutra. Segundo, la medida o la práctica pone en una situación desaventajada un grupo de personas protegido. Es el segundo criterio de la discriminación indirecta el que difiere de la discriminación directa: el análisis de la discriminación no se focaliza sobre la existencia de un trato diferencial sino sobre los efectos diferenciales». Corte Constitucional. Sentencia T-909 de 2011. 
sexual, pertenencia étnica, estatus socioeconómico, y la discapacidad, entre $\operatorname{otros}^{13}$.

En el examen de la imposición del IVA a los elementos de higiene femenina, la Corte adicionalmente acogió los criterios propuestos por Barnett y Grown, centrados en la necesidad de reparar en cuatro hechos comunes que determinan el impacto de las políticas tributarias en la igualdad de las mujeres. Estos se corresponden con las diferencias de género en nuestra sociedad respecto a «(i) el empleo remunerado, incluido el trabajo formal e informal, salarios y segregación ocupacional; (ii) el porcentaje de trabajo no remunerado que realizan las mujeres como el doméstico, el cuidado de niños, personas enfermas y de la tercera edad; (iii) los gastos de consumo; y (iv) los derechos de propiedad ${ }^{14}$.

De acuerdo con lo planteado por el Alto Tribunal, dichos criterios resultan fundamentales, ya que permiten develar la forma en que ciertos tributos, al no gravar la capacidad adquisitiva de las personas asumiendo que el trato igual para todas resulta equitativo, como ocurre con el IVA, en realidad pueden tener implicaciones diferenciales para las mujeres al recaer sobre bienes insustituibles para ellas, lo cual influye directamente en el goce de sus derechos a la salud y dignidad.

Así pues, haciendo uso de estas herramientas y teniendo en cuenta el contexto de la disposición que grava con el IVA a las toallas y tampones higiénicos, y bajo el entendido de que estos elementos son bienes irremplazables para la población femenina — pues no existen otros que puedan suplirles y se constituyen en elementos de primera necesidad-, la Corte determinó que dicha tarifa no es razonable en nuestro orden constitucional. Ello por cuanto, en primer lugar, correspondía al legislador la carga de determinar por qué persistía la imposición del gravamen a estos elementos en la reforma tributaria; en segundo lugar, debido a que no existen políticas públicas que compensen las barreras de acceso a estos elementos a las mujeres con menos recursos económicos; y finalmente, porque la capacidad adquisitiva de la población femenina está supeditada por, al menos, cuatro "presupuestos de desventaja en la parti-

$13 \mathrm{Al}$ respecto, el Comité de la CEDAW en su Recomendación General 28 indicó que esta discriminación "puede darse simultáneamente con otros factores tales como raza, etnia, religión o creencia, salud, status, edad, clase, casta y orientación sexual. El enfoque interseccional obliga a los Estados a adoptar medidas diferentes para los distintos grupos poblacionales de mujeres discriminadas».

14 Barnett, K. y Grown, C. (2004), en Grown, C. y Valodia, I. Taxation and Gender Equity. A comparative analysis of direct and indirect taxes in developing and developed countries (p. 4 y ss.) (2010). Disponible en: https://bit.ly/2OaqSgV. Citado en sentencia C-117 de 2018. 
cipación económica que generan que este tipo de medidas tengan un impacto desproporcionado para ellas, en especial para las mujeres más pobres».

En consecuencia, la Corte concluyó que el tributo,

[...] al gravar las toallas higiénicas y los tampones, viola el principio de equidad tributaria pues impone barreras al acceso de tecnologías que actualmente permiten el pleno ejercicio del derecho a la dignidad de las mujeres, su participación mujeres en la vida pública, al igual que la protección de los derechos a la salud, a la educación y al trabajo y a la dignidad humana. En ese sentido, la disposición tiene un impacto desproporcionado para las mujeres con baja capacidad adquisitiva que no tienen la opción de reemplazarlos con otros bienes similares que resulten más económicos o no estén gravados ni generen riesgos para la salud.

A partir de las anteriores reflexiones, ordenó incluir los elementos de higiene menstrual dentro de los bienes exentos de tributos.

Este fallo es muy significativo, pues a partir de este Colombia entró dentro del aún restringido listado de países que han comprendido que el deber de avanzar en la garantía del derecho a la igualdad de las mujeres y las niñas necesariamente pasa por la adopción de medidas de justicia tributaria y económica.

Gravar los elementos de higiene menstrual bienes implica reducir la posibilidad de acceso a los mismos, en particular para las mujeres y niñas con menos capacidad adquisitiva. Lo anterior tiene mayor relevancia si se tienen en cuenta las condiciones estructurales de desigualdad entre hombres y mujeres que persisten en el país, en las que estas continúan siendo la población con menos recursos económicos, lo cual tiene trascendentales consecuencias para su desarrollo.

La utilización de tecnologías para la atención del flujo menstrual, como manifestación biológica natural de la población femenina, implica una inversión cuantiosa de parte de las mujeres a lo largo de sus vidas, debiendo destinar una parte considerable de sus ingresos a ese fin, independientemente de su capacidad adquisitiva. Esto es muy importante dado que la población femenina continúa siendo el sector del país con menos recursos económicos, la cual, además de tener que suplir sus otras necesidades básicas, debe incurrir en este gasto. De allí que someterlas a sufragar costos adicionales, desproporcionados e injustificados de los productos de higiene menstrual tiene un impacto directo en sus derechos fundamentales, respecto de los cuales se ha advertido de que el Estado tiene el especial deber de observar las diferencias de hecho existentes en la sociedad, para no profundizarlas con medidas impositivas.

Un factor aparentemente intrascendente como la posibilidad de comprar dichos elementos de higiene impacta directamente en sus derechos reproduc- 
tivos $^{15}$ —en su componente de prevención de las enfermedades del aparato reproductor femenino- ${ }^{16}$, así como en los derechos a la dignidad, salud, educación y participación social, e incrementa la brecha que existe respecto a las garantías de las que goza la población masculina para ejercerlos, lo cual desconoce los múltiples compromisos que tiene el Estado colombiano para eliminar esa distancia. De esta manera, impedir que el sistema tributario sea un factor adicional de discriminación constituye un paso adelante en la garantía de los derechos de las mujeres y las niñas que evidencia la necesidad de atender todos los escenarios sociales que tienen incidencia en ello, como un presupuesto para el éxito en su protección.

\section{UNO ATRÁS: EL CAMBIO DE PRECEDENTE SOBRE ESTABILIDAD LABORAL REFORZADA A MUJERES GESTANTES}

En la sentencia SU-075 de 2018, la Sala Plena de la Corte Constitucional estudió las acciones de tutela interpuestas por tres mujeres en estado de gestación quienes consideraron vulnerados sus derechos fundamentales al trabajo, a la estabilidad laboral reforzada, a la salud y a la igualdad, tras la decisión unilateral de sus empleadores de dar por terminados sus contratos de trabajo.

Las empresas para las cuales laboraban argumentaron desconocer su particular condición al momento de desvincularlas, razón por la cual estimaban que no les correspondía garantizar la continuación en sus empleos o sufragar los gastos correspondientes al Sistema de Seguridad Social en Salud para que gozaran de la licencia de maternidad.

Así pues, la Corte consideró que el problema jurídico que debía resolver era si las empresas privadas accionadas desconocieron los derechos fundamentales de las mujeres gestantes al finalizar unilateralmente sus contratos de trabajo, aun cuando para ese momento desconocían dicho estado de las trabajadoras.

15 Recientemente, la Corte Constitucional, en la sentencia T-398 de 2019, determinó que los derechos fundamentales, la dignidad humana y los derechos sexuales y reproductivos de las mujeres se ven comprometidos cuando no se tiene acceso efectivo a bienes relacionados con la gestión de la higiene menstrual.

16 Al respecto, ver los elementos de la salud reproductiva identificados por la Corte Constitucional en las sentencias T-627 de 2012, C-093 de 2018 y la SU-096 de 2018. 
Aunque, en su pronunciamiento, el Alto Tribunal mantuvo el precedente general en relación con la estabilidad laboral reforzada de mujeres gestantes, decidió modificar su posición respecto de los deberes de la entidad empleadora cuando desvincula a una trabajadora, sin conocer el estado de la misma. La pauta establecida anteriormente en la sentencia SU-070 de 2013 imponía, en esos eventos, la obligación a cargo de las entidades contratantes de pagar las cotizaciones al Sistema General de Seguridad Social en Salud hasta el momento del parto y, en algunos casos, también la licencia de maternidad.

No obstante, a partir del estudio de estos casos estimó que dicha regla era «contraria a los valores, objetivos, principios y derechos en los que se funda el ordenamiento jurídico, porque establecía una carga desproporcionada para el empleador pese a que su actuación no había sido motivada en criterios discriminatorios». De esta manera llegó a la conclusión de que esta exigencia a los empleadores desincentivaba la contratación de mujeres en edad reproductiva, lo cual podría derivar en una mayor discriminación para aquellas en el ámbito laboral.

La Corte consideró que con su decisión no se desamparaba a las mujeres cuya gestación era desconocida por el empleador al momento de dar por terminada su relación laboral, ya que el ordenamiento jurídico cuenta con otras medidas para garantizar los derechos de la mujer y del niño o niña que está por nacer. Así, sobre su derecho a la salud, hizo mención a la posibilidad que tienen estas de ser beneficiarias de otro familiar afiliado al Régimen Contributivo y recordó el deber legal que recae sobre el Régimen Subsidiado de atenderles cuando no cuentan con recursos para tener la calidad de cotizantes independientes. Frente al mínimo vital, señaló que el ordenamiento jurídico cuenta con varias alternativas de protección tales como el subsidio alimentario a cargo del ICBF consagrado en la Ley 100 de 1993 y los mecanismos de subsidio al desempleo introducidos por la Ley 1636 de 2013. En palabras del Alto Tribunal, «[d]e este modo, se desarrolla el artículo 43 Superior, el cual dispone que la mujer "[d]urante el embarazo y después del parto gozará de especial asistencia y protección del Estado, y recibirá de éste subsidio alimentario si entonces estuviere desempleada o desamparada"».

Igualmente, destacó que su decisión no implicaba la determinación de una tarifa legal para demostrar que el empleador sabía de la condición de gestante de la mujer trabajadora, pues existe libertad probatoria para ello. En conclusión, a partir de este fallo en Colombia, «cuando se demuestra en el proceso de tutela que el empleador no conoce acerca del estado de gestación de la trabajadora en el momento del despido, con independencia de que se haya aducido una justa causa, no hay lugar a la protección derivada de la estabilidad laboral reforzada». 
A nuestro juicio, esta sentencia implica uno de los retrocesos más sensibles de la jurisprudencia constitucional de los últimos tiempos frente a los derechos de las mujeres, por sus implicaciones en su acceso al ámbito laboral - respecto del que persisten múltiples retos para constituirse en un espacio efectivamente incluyente- y sobre su autonomía reproductiva ${ }^{17}$. Esto es sumamente lesivo, si se tiene en cuenta que el trabajo tiene profundas consecuencias para el desarrollo humano, al permitir llevar a cabo el proyecto de vida que cada quien elige y posibilitar la independencia económica, la cual, como decía Simone de Beauvoir, para las mujeres es especialmente significativa, por ser la primera condición para acceder también a una independencia interior, moral y mental, y por constituir la manera más eficaz de evadir la opresión masculina ${ }^{18}$. Legitimar el despido cuando no se había reportado el estado de gestación confirma el temor de las mujeres de que la decisión de ser madres puede frenar, retroceder o truncar su crecimiento y desenvolvimiento profesional o laboral.

Tal como fue expresado en varios salvamentos de voto ${ }^{19}$, esta sentencia parte de planteamientos sumamente problemáticos que alteran el concepto de fuero de maternidad y traen como consecuencia la desprotección de quienes deciden ser mamás en nuestro país, así como de sus hijos o hijas. Tanto la justificación para generar el cambio jurisprudencial como varios de sus racionamientos son cuestionables.

En primer lugar, el fundamento para redefinir la regla a aplicar en aquellos casos en los que el empleador desconocía del estado de gestación de la trabajadora consiste en la exposición de diversos estudios que darían cuenta de que la mayor tasa de desempleo es ocupada principalmente por las mujeres y de las especiales dificultades que enfrentan aquellas en edad reproductiva a la hora de emplearse. Así pues, las cifras podrían sustentar la tesis de que medidas afirmativas como la licencia de maternidad y la prohibición de despedir a una gestante implican un desincentivo para su contratación, debido a los sobrecostos que ello implica. Sin embargo, es fácil advertir que eso no tiene una incidencia clara y directa sobre el hecho de que el contratante conociera del

17 En cuanto a la autodeterminación reproductiva, la Corte ha señalado que de él se deriva «el derecho a estar libres de todo tipo de interferencias en la toma de decisiones reproductivas, incluida la violencia física y psicológica, la coacción y la discriminación, pues no se deben sufrir tratos desiguales injustificados por razón de las decisiones reproductivas, sea que se decida tener descendencia o no». T-627 de 2012. Reiterada en la sentencia T-274 de 2015.

18 De Beauvoir, Simone (s. f.). Ver en: https://bit.ly/34o1euM.

19 Específicamente, los realizados por la magistrada Diana Fajardo Rivera (2018) y el magistrado Alberto Rojas Ríos (2018). 
estado de la trabajadora previamente a dar por terminado el vínculo laboral, lo cual es en realidad el objeto de variación del precedente.

En segundo lugar, vale la pena también controvertir un par de razonamientos complejos sobre el concepto de estabilidad laboral reforzada de mujeres gestantes, por las conclusiones que el Alto Tribunal deriva a partir de aquellos. Por un lado, sostiene que esta es una acción afirmativa en respuesta a la discriminación histórica enfrentada por las mujeres a causa de su rol en la reproducción humana. De esta manera, cuando la mujer es despedida por el empleador sin que conociera previamente de su estado, no la estaría discriminando, y, por ello, no es dable asignarle una responsabilidad de protección, que, en todo caso, debe recaer en el Estado y no en los particulares.

Dicho planteamiento presenta al menos tres aspectos debatibles sobre la estabilidad laboral reforzada. El primero es considerar que esa es su única y principal finalidad, pues, aunque efectivamente es una medida que pretende hacerle frente a la discriminación, tiene como propósito mayor el volver compatible la maternidad con el trabajo, de suerte que la decisión de convertirse en madre no represente para las mujeres su exclusión del mercado laboral. Atacar las distintas formas de marginación busca garantizar a los grupos que tradicionalmente la han padecido su inclusión, y a partir de ella, el goce de sus derechos.

El segundo es que desconoce que la estabilidad laboral reforzada es una protección jurídica objetiva en favor de las mujeres gestantes, independientemente de que ellas sepan de su estado o se lo hayan comunicado a su empleador. Es decir, nuestra Constitución prevé una garantía para quienes se encuentran en estado de gestación sin haberlo supeditado a determinadas condiciones, como, por ejemplo, su publicidad, sino al exclusivo hecho de encontrarse en ese estado biológico.

El tercero, directamente ligado al anterior, es ignorar que el estado de gestación no es siempre susceptible de diagnóstico médico desde el momento de su inicio debido a las particulares condiciones de cada mujer. Así pues, la protección dada a aquellas por nuestro ordenamiento jurídico debe otorgarse por la sola existencia de la gestación y no por la posibilidad de acreditación de la misma. Pasar por alto por esto resulta en la desprotección de aquellos a quienes no es legítimo exigirles el conocimiento de una situación respecto de la cual ni siquiera las herramientas técnicas disponibles en el país pueden otorgar certeza.

Por otra parte, el segundo razonamiento que resulta cuestionable es el alcance que otorga la Corte a la inescindibilidad de los fundamentos de la estabilidad laboral reforzada, al entender la reducción o disolución de aquellos al principio de no discriminación. La interdependencia de tales fundamentos implica que su comprensión debe hacerse de manera relacional y en diálogo 
permanente, pero de ninguna manera que se diluyan exclusivamente en la salvaguarda del derecho a la igualdad en el trabajo. Con esta tesis, se habría abandonado la sólida base dada al fuero de maternidad desde distintos e importantes preceptos constitucionales, entre los que se encuentran el mínimo vital, la vida del que está por nacer y la relevancia de la familia.

Ahora bien, se hace necesario también dar cuenta de que la decisión no tiene solamente consecuencias sobre las mujeres sino sobre sus hijos e hijas. A partir de esta, se produce una diferencia injustificada respecto de los derechos de los niños y las niñas, al depender del hecho de que sus madres tuvieran la posibilidad de dar a conocer su estado de gestación a su empleador antes de ser despedidas. Así, un grupo de ellos se verá sometido a las difíciles condiciones que enfrenta su progenitora cuando no goza de los beneficios de la respectiva licencia y debe acudir a las circunstancias a las que se ve sometida por el desempleo.

Asimismo, vale la pena llamar la atención sobre la intromisión que representa ello en la intimidad de cada mujer al obligarle a reportar su estado cuanto antes, so pena de no ser protegida, cuando este evento representa un cambio en su vida que debe ser asumido, manejado y comunicado acorde con sus deseos y a lo que implique ello en sus proyectos personales.

Finalmente, no puede pasarse por alto que la salvedad que hace la Corte sobre que su decisión no representa una desprotección a las mujeres gestantes, pues estas pueden ser beneficiarias de las medidas asistenciales contenidas en la Ley 1636 de 2013 y en el art. 166 de la Ley 100 de 1993, carece de sentido ya que las disposiciones en mención no fueron diseñadas para garantizar la dimensión integral del derecho fundamental a la estabilidad laboral reforzada y tienen limitaciones, al imponer requisitos para su acceso, que, por supuesto, no todas las mujeres están en posibilidad de acreditar.

Como se observa, la desprotección a la maternidad derivada de este pronunciamiento significa un paso atrás en los derechos de las mujeres con graves implicaciones para su autonomía reproductiva, al permitir su despido cuando no dieron a conocer previamente el estado de gestación a su empleador, lo que en últimas resulta en una interferencia ilegítima en la toma de su decisión sobre tener descendencia, así como respecto de sus derechos laborales.

\section{CONCLUSIONES}

La jurisprudencia constitucional ha jugado un importante papel en la garantía de los derechos fundamentales de las mujeres y las niñas al adoptar decisiones que propenden por erradicar la violencia y exclusión en su contra. 
Durante 2018, tres pronunciamientos se destacaron por su impacto en los derechos reproductivos de la población femenina en el país. Se trata de dos positivos y uno negativo. En primer lugar, la sentencia SU-096 de 2018 mantuvo más de una década de protección a la interrupción voluntaria del embarazo. El estudio del caso en cuestión dio cuenta de la persistencia de los obstáculos para el ejercicio de dicho derecho fundamental pese a la claridad de los criterios para la práctica del procedimiento correspondiente, dictados en múltiples fallos precedentes. Insistir en su protección y ordenar la regulación correspondiente para garantizar su goce constituye un importante avance que incide en el goce de mejores condiciones de vida para la población femenina.

En segundo lugar, la sentencia C-117 de 2018, relativa a la exención tributaria a los elementos para la higiene menstrual, a partir de la que Colombia se sumó a los países que han comprendido que el deber de avanzar en la garantía del derecho a la igualdad de las mujeres y las niñas necesariamente implica la adopción de medidas de justicia tributaria y económica.

La posibilidad de comprar toallas y tampones higiénicos impacta directamente en los derechos reproductivos, a la dignidad, salud, educación y participación social de la población femenina. Impedir que el sistema tributario sea un factor adicional de discriminación en su contra representa un paso adelante en su protección, lo que evidencia la necesidad de atender todos los escenarios que puedan tener incidencia en dicho propósito.

Finalmente, el retroceso estuvo dado a partir de la sentencia SU-075 de 2018, que inusitadamente dio un paso atrás en la estabilidad laboral reforzada de mujeres gestantes, ya que, en este pronunciamiento, el Alto Tribunal decidió modificar su posición respecto de los deberes de la entidad empleadora cuando desvincula a una trabajadora sin conocer el estado de gestación de la misma, a partir de lo cual limitó su amparo.

Dichos fallos evidencian que el avance en la garantía de los derechos de la población femenina sigue siendo un proceso lento, cuya consolidación representa un desafío constante para la jurisprudencia constitucional. Recorrer el camino hacia la igualdad implica continuar dando pasos decididos en la garantía de los derechos reproductivos de la población femenina por su impacto en el desarrollo de miles de mujeres y niñas.

\section{Apéndice legislativo}

Corte Constitucional de Colombia. (2011). Sentencia T-909 de 2011. Bogotá: Corte Constitucional

Corte Constitucional de Colombia. (2012). Sentencia T-627 de 2012. Bogotá: Corte Constitucional 
Corte Constitucional de Colombia. (2013). Sentencia SU-070 del 2013. Bogotá: Corte Constitucional.

Corte Constitucional de Colombia. (2017). Sentencia T-735 de 2017. Bogotá: Corte Constitucional.

Corte Constitucional de Colombia. (2017). Sentencia C-005 del 2017. Bogotá: Corte Constitucional.

Corte Constitucional de Colombia. (2018). Sentencia T-338 de 2018. Bogotá: Corte Constitucional.

Corte Constitucional de Colombia. (2018). Sentencia SU-075 del 2018. Bogotá: Corte Constitucional.

Corte Constitucional de Colombia. (2018). Sentencia SU-096 del 2018. Bogotá: Corte Constitucional.

Corte Constitucional de Colombia. (2018). Sentencia C-117 del 2018. Bogotá: Corte Constitucional.

Corte Constitucional de Colombia. (2019). Sentencia T-398 de 2019. Bogotá: Corte Constitucional.

\section{Bibliografía}

Barnett, K. y Grown, C. (2004). Gender impacts of Government Revenue Collection: The Case of Taxation. London: Commonwealth Secretariat.

Comité de Derechos Económicos, Sociales y Culturales (2017). Observaciones finales sobre el sexto informe periódico de Colombia. (E/C.12/COL/CO/6).

Comité para la Eliminación de la Discriminación en contra de la Mujer (2010). Recomendación General No 28 relativa al artículo 2 de la Convención sobre la eliminación de todas las formas de discriminación contra la mujer. (CEDAW/C/GC/28).

De Beauvoir, S. (s. f.). El segundo sexo. Buenos Aires: Siglo XX Editores. Disponible en: https://bit.ly/2NDbQir.

Fajardo Rivera, D. (2018). Salvamento a la sentencia SU-075 de 2018. Bogotá: Corte Constitucional.

La Mesa por la Vida y la Salud de las Mujeres (2017). Barreras de acceso a la interrupción voluntaria del embarazo en Colombia. Disponible en: https://bit.ly/2ovEFow.

Pardo Schlesinger, C. (2018). Salvamento de voto a la sentencia SU-096 del 2018. Bogotá: Corte Constitucional.

Rojas Ríos, A. (2018). Salvamento a la sentencia a la sentencia SU-075 de 2018. Bogotá: Corte Constitucional. 